\title{
Association Between Duration of Constipation and Frequency of Urinary Tract Infection in Children
}

\author{
Pedram Ataee ${ }^{1}$, Bahare Taleshi ${ }^{2}$, Alireza Eskandarifar ${ }^{3}$, Bijan Nuri ${ }^{4}$, Rama Naghshizadian ${ }^{2}$, Armen \\ Malekian Taghi ${ }^{5}$ and Kambiz Eftekhari (iD) 6," \\ ${ }^{1}$ Liver and Digestive Research Center, Research Institute for Health Development, Department of Pediatric, Kurdistan University of Medical Sciences, Sanandaj, Iran \\ ${ }^{2}$ Kurdistan University of Medical Sciences, Sanandaj, Iran \\ ${ }^{3}$ Pediatric Department, Faculty of Medicine, Kurdistan University of Medical Sciences, Sanandaj, Iran \\ ${ }^{4}$ Social Determinants of Health Research Center, Research Institute for Health Development, Kurdistan University of Medical Sciences, Sanandaj, Iran \\ ${ }^{5}$ Pediatric Department, Tehran University of Medical Sciences, Sanandaj, Iran \\ ${ }^{6}$ Pediatric Gastroenterology and Hepatology Research Center, Tehran University of Medical Sciences, Tehran, Iran \\ "Corresponding author: Associate Professor of Pediatric Gastroenterology, Department of Pediatric, Bahrami Children's Hospital, Tehran, Iran. Email: \\ dr_k_eftekhary@yahoo.com
}

Received 2020 May 03; Revised 2020 May 20; Accepted 2020 June 01.

\begin{abstract}
Background: Constipation and Urinary Tract Infection (UTI) are common problems in children. The gastrointestinal tract and the urinary system are related together anatomically and functionally. Constipation is one of the possible causes of UTI and its recurrence.

Objectives: The purpose of this study was to evaluate the association between the duration of constipation and the frequency of upper and lower UTI in children in Sanandaj.

Methods: A descriptive-analytical study was performed on children with chronic constipation aged less than 12 years, referring to the Pediatric Gastroenterology Clinic of Besat Hospital in Sanandaj in 2018-2019. Urine analysis and culture were performed for all the patients. Data were recorded in separate questionnaires.

Results: There were 220 children in this study. Most cases of constipation and UTI belonged to the group of 3-6 years. Constipation was more common in boys and UTI in girls. Lower UTI was more common than upper UTI. Besides, $45 \%$ of the patients had constipation for less than a year. There was no significant relationship between the duration of constipation and the prevalence and type of UTI (upper or lower) $(\mathrm{P}=0.405, \mathrm{P}=0.911)$.

Conclusions: Urinary tract infection was common in children with chronic constipation. There was no relationship between the duration of constipation and the frequency and type of UTI.
\end{abstract}

Keywords: Childhood, Chronic Functional Constipation, Urinary Tract Infection

\section{Background}

Constipation refers to bowel movements fewer than twice a week. According to the definition, chronic constipation should have two or more of the following symptoms for at least three months: Excessive strain during a bowel movement, stiffness in stool consistency, feeling incomplete excretion after a bowel movement, and defecation less than twice a week (1-3). Various studies have shown the interaction between the function of the urinary system and the intestine. The association between the bladder and intestine is evident through the presence of urinary symptoms in patients with irritable bowel syndrome and the presence of intestinal symptoms in patients with acute cystitis. Gastrointestinal disorders such as constipation can lead to lower urinary tract symptoms, including bladder hyperactivity (4). The embryonic origin of the bladder and rectum is the same, and their autonomic and somatic innervations are similar. Due to the proximity of these two organs, the dysfunction of one of them can affect the other. Therefore, defecation disorders can be associated with urinary tract problems.

Studies in children have shown that constipation is associated with urinary tract problems, including infection, urinary incontinence, vesicoureteral reflux, and urinary tract obstruction. The underlying pathophysiology of these findings has not yet been elucidated (5). A study showed that an increase in rectal diameter is associated with a high frequency of urinary tract infections (6). Another author depicted that the treatment of constipa- 
tion resulted in significant urinary control (7). Some researchers found that the prevalence of constipation was $8 \%$ in children with UTI, and the prevalence of recurrent UTI was $67.8 \%$ in children with constipation. A significant relationship was found between recurrent UTI and constipation (8). Previous publications have not indicated any association between constipation duration and the frequency of UTI and its upper and lower types (cystitis and pyelonephritis).

\section{Objectives}

The purpose of this study was to evaluate the link between constipation duration and urinary tract infection.

\section{Methods}

This descriptive-analytical study utilized a simple random sampling method. The population consisted of children with chronic constipation aged 1 - 12 years who referred to the Pediatric Gastroenterology Clinic of Besat Hospital of Sanandaj during one year (2018 - 2019). The inclusion criteria included children with chronic constipation aged 1-12 years (based on the Rome IV criteria (9)). The exclusion criteria were children with organic constipation and/or previous use of antispasmodics or antibiotics, and patients who had a history of anorectal surgery or anatomical disorders of the urinary tract.

\subsection{Intervention}

Informed consent was obtained from children or their parents. The questionnaires were filled out by parents. Each questionnaire consisted of demographic information including age, sex, place of residence, duration of chronic constipation, and history or presence of urinary symptoms (including dysuria, frequency, hematuria, abdominal pain, flank pain, and fever). All children were examined for UTI. For this purpose, a sterile urine sample was sent for analysis and culture. Urinary tract infection was considered when there were more than $10^{5}$ colonies of one type of a pathogen or $10^{4}$ colonies in symptomatic children with active urine (10). We considered fever as the main criterion for distinguishing upper and lower urinary tract infections $(11,12)$. Children with UTI were referred to the Nephrology Clinic for treatment. Appropriate treatment for chronic constipation was also prescribed. The study was approved by the Ethics Committee of Kurdistan University of Medical Sciences (Ethical Number: IR.MUK.REC.1397/162).

\subsection{Statistic Analysis}

Data were analyzed using SPSS V. 22 software and descriptive statistics. After determining the prevalence of constipation and UTI and their 95\% confidence intervals, the chi-square and independent $t$-tests were used to evaluate the study hypotheses. P values of less than 0.05 were considered statistically significant.

\section{Results}

In this study, 220 patients participated. There were $54.55 \%(n=120)$ male patients. Most children were in the age group of 3 - 6 years. Besides, $83 \%(n=183)$ were urbanites. In terms of duration, $45 \%$ suffered from constipation for less than a year, 33\% for one to two years, and about $22 \%$ for more than two years (Table 1$)$. Moreover, $16 \%(n=35)$ had concurrent UTI. Of them, $46 \%$ suffered from constipation for less than one year, $26 \%$ for one to two years, and $28 \%$ for more than two years. There was no significant relationship between the duration of constipation and the frequency of UTI (P value $=0.450)$ (Table 1$)$. Among children with UTI, the ratio of girls to boys was $2: 1$ and there was a significant relationship between gender and UTI $(\mathrm{P}$ value $=$ 0.010). The highest number of UTI was encountered in the group of 3 - 6 years. There was no significant relationship between age and UTI (P value $=0.528)$. Among children with UTI, about $46 \%(n=16)$ had upper UTI (pyelonephritis) and 54\% ( $n=19)$ had lower UTI (cystitis). There was no significant relationship between the duration of constipation and the type of UTI (P value $=0.911)$. The details on the type of UTI and duration of chronic constipation are presented in Table 2. The demographics of patients with each type of UTI and duration of chronic constipation are summarized in Table 2 . There was no significant relationship between the type of UTI and gender and age (P value $=0.909$, P value $=0.476$, respectively). The duration of chronic constipation, frequency of UTI, and age distribution are summarized in Tables 1 and 2. The place of residence had no significant relationship with constipation, UTI, and types of UTI ( $\mathrm{P}$ value $=0.808$ and $\mathrm{P}$ value $=1.000$, respectively) (Tables 1 and 2). The most common symptoms of UTI were frequency (14.55\%), dysuria (12.73\%), abdominal pain (7.73\%), and fever (7.27\%). The flank pain, hematuria, nausea, and vomiting were rare. Urinalysis was abnormal in $14.5 \%$ of the patients, and urine culture was positive in $8.2 \%$.

\section{Discussion}

Constipation is a common gastrointestinal problem in children that can impose huge costs on society. This problem is a major complaint in 3\% of referrals to physicians' 


\begin{tabular}{|c|c|c|c|}
\hline Variable & $\begin{array}{c}\text { Chronic } \\
\text { Constipation, No. (\%) }\end{array}$ & UTI, No. (\%) & P Value \\
\hline Age group, mo & & & 0.528 \\
\hline $1-36$ & $43(19.55)$ & $8(18.60)$ & \\
\hline $37-72$ & $119(54.09)$ & $16(13.45)$ & \\
\hline$>72$ & $58(26.35)$ & $11(18.97)$ & \\
\hline Sex & & & 0.010 \\
\hline Male & $120(54.55)$ & $12(10.00)$ & \\
\hline Female & $100(45.45)$ & $23(23.00)$ & \\
\hline Place of living & & & 0.808 \\
\hline Urban & $183(83.18)$ & $30(16.39)$ & \\
\hline Rural & $37(16.82)$ & $5(13.51)$ & \\
\hline \multicolumn{4}{|c|}{$\begin{array}{l}\text { Duration of } \\
\text { constipation, mo }\end{array}$} \\
\hline $1-24$ & $99(45.00)$ & $16(16.16)$ & 0.405 \\
\hline $25-48$ & $73(33.18)$ & $9(12.33)$ & 0.528 \\
\hline$>48$ & $48(21.825)$ & $10(20.83)$ & \\
\hline
\end{tabular}

\begin{tabular}{|c|c|c|c|}
\hline Variable & Upper UTI, No. (\%) & Lower UTI, No. (\%) & PValue \\
\hline Age group, mo & & & 0.909 \\
\hline $1-36$ & $3(37.50)$ & $5(62.50)$ & \\
\hline $37-72$ & $8(50.00)$ & $8(50.00)$ & \\
\hline$>72$ & $5(45.45)$ & $6(54.55)$ & \\
\hline Sex & & & 0.476 \\
\hline Male & $4(33.33)$ & $8(66.67)$ & \\
\hline Female & $12(52.17)$ & $11(47.83)$ & \\
\hline Place of living & & & 1.000 \\
\hline Urban & $14(46.67)$ & $16(53.33)$ & \\
\hline Rural & $2(40.00)$ & $3(60.00)$ & \\
\hline $\begin{array}{l}\text { Duration of } \\
\text { constipation, mo }\end{array}$ & & & 0.911 \\
\hline $1-24$ & $8(50.00)$ & $8(50.00)$ & \\
\hline $25-48$ & $4(44.44)$ & $5(46.55)$ & \\
\hline$>48$ & $4(40.00)$ & $6(60.00)$ & \\
\hline
\end{tabular}

clinics and $10 \%-25 \%$ of referrals to pediatric gastroenterologists. It is, therefore, a major problem for both patients and families (13). Considering the high prevalence of UTI and constipation in children, as well as the complications of UTI on the developing kidney, it is important to investigate the causative and exacerbating factors. On the other hand, constipation is one of the possible contributing factors to the development and recurrence of UTI. However, there are controversial results about the role of bowel dysfunction in urinary disorders such as urinary reflux, UTI, and urinary incontinence. We found that the frequency of constipation was highest in the group of $3-6$ years (54.09\%). Hakimzadeh et al. showed that most cases of constipation were in the group of $2-7$ years $(84.47 \%)$ (8). In the Sampaio study, the mean age of the patients was nine years (7 - 11 years) (14). This difference may be related to the difference in the participants' age in the studies ( 1 - 12 years vs. 5 - 17 years). The most common age group of constipation patients was between one and five years in Sarvari's study (15). According to our results, about $16 \%$ of children with chronic constipation also had UTI. This rate was reported as $67.8 \%$ by Hakimzadeh et al. (8) and $27 \%$ by Sampaio et al. (14). These studies suggested a significant association between UTI and constipation. Sampaio demonstrated that children with constipation were about seven times more likely to have inferior UTI, and there was a significant association between the two diseases. Sarvari et al. reported a 13.3\% UTI prevalence in children with constipation (15). According to our results, there was no relationship between the frequency of UTI, its types (upper or lower infection), and the duration of constipation (P value $=0.450$ ) . Since no previous studies have examined this relationship, we could not compare our findings with others. More than half of the patients in our study were boys, while in other studies, constipation was more common in girls $(8,14,16)$. This difference may be due to differences in race and culture. In some areas, boys are more than girls. The majority of patients in our study were from urban areas. The justification for this discrepancy is that urbanites are more likely to see a doctor for treatment than rural people. The most common clinical manifestations of UTI were frequency, dysuria, abdominal pain, and fever. Dehghani et al. also reported that the most common symptoms were dysuria and frequency (16). In the Sarvari et al. study, the most common manifestations of UTI were fever, dysuria, and frequency (15). The results of urinalysis and culture vary among different studies. Dehghani et al. exhibited abnormal urine analysis in $8.3 \%$ and positive urine culture in $5.8 \%$ of patients (16). In the Sarvari et al. study, the urinary culture was positive in $13.3 \%$ (15). We found that urinalysis was abnormal in $14.55 \%$, and urine culture was positive in $8.18 \%$. We noted that lower UTI was more common than upper UTI. This may be due to the anatomical association between the bladder and rectum, so cystitis and lower UTIs were more common in these children. In our study, UTI was more common in girls. Many studies are consistent with our results and showed that UTI was more frequent in girls $(8,15)$. This can be due to the shortness of urethra in girls and the anatomical abnormalities such as labia majora adhesion. The UTI was mostly found in the age group of $3-6$ 
years. Hakimzadeh et al. depicted the highest incidence of UTI in the age group of $2-7$ years (8).

\subsection{Conclusion}

Constipation and UTI were more common in the age group of 3 - 6 years. Constipation was more common in boys and UTI in girls. The prevalence of UTI was about $16 \%$ in children with constipation, with lower UTI being more common. Most of these children had constipation for less than a year. The duration of constipation had no significant relationship with the prevalence and type of UTI.

\section{Acknowledgments}

We thank the honorable research vice-chancellor of the University and the Faculty of Medicine of Kurdistan University of Medical Sciences, and the staff of Besat Hospital, who assisted us in this research. Also, we appreciate the cooperation of Dr. Diana Diaz with writing this article.

\section{Footnotes}

Authors' Contribution: Study concept and design: Pedram Ataee, Bahare Taleshi, Bijan Nuri, Rama Naghshizadian, and Kambiz Eftekhari. Acquisition of data: Pedram Ataee, Bahare Taleshi, Alireza Eskandarifar, Rama Naghshizadian, and Armen Malekiantaghi. Analysis and interpretation of data: Pedram Ataee, Bahare Taleshi, Bijan Nuri, and Kambiz Eftekhari. Drafting of the manuscript: Pedram Ataee, Alireza Eskandarifar, Bijan Nuri, Armen Malekiantaghi, and Kambiz Eftekhari. Critical revision: Pedram Ataee, Bahare Taleshi, Rama Naghshizadian, Armen Malekiantaghi, and Kambiz Eftekhari.

Conflict of Interests: The authors have no conflicts of interest to disclose.

Ethical Approval: The study was approved by the Ethics Committee of Kurdistan University of Medical Sciences (Ethical Number: IR.MUK.REC.1397/162).

Funding/Support: No external funding was secured for this study.

Informed Consent: Informed consent was obtained from children or their parents.

\section{References}

1. Sood MR. Constipation in Infants and Children: Evaluation. UpToDate; 2020.

2. Kuizenga -Wessel S, Benninga MA. Functional Constipation and Incontinence. In: Wyllie R, Hyams JS, Kay M, editors. Pediatric gastrointestinal and liver disease. 5th ed. Philadelphia: Elsevier Saunders; 2016. p.124-36.

3. Tobin V. Obsessive-Compulsive Disorder in Children and Adolescents. J Psychosoc Nurs Ment Health Serv. 2018;56(3):15-8. doi: 10.3928/02793695-20180219-02. [PubMed: 29505086].

4. Kaplan SA, Dmochowski R, Cash BD, Kopp ZS, Berriman SJ, Khullar V. Systematic review of the relationship between bladder and bowel function: implications for patient management. Int J Clin Pract. 2013;67(3):205-16. doi: 10.1111/ijcp.12028. [PubMed: 23409689].

5. Averbeck MA, Madersbacher H. Constipation and LUTS - how do they affect each other? Int Braz J Urol. 2011;37(1):16-28. doi: 10.1590/s167755382011000100003. [PubMed: 21385476].

6. Alaee A, Karami H, Shahmohammadi S, Mehrara Z. Relationship between rectum diameter and constipation with urinary tract infections in children. J Gorgan Univ Med Sci. 2014;16(2):57-61.

7. Shahraki T, Sadeghi-Bojd S, Shahraki M. Effect of Constipation Treatment on Urinary Tract Dysfunction in Children. Zahedan J Res Med Sci. 2010;12(3). e94293.

8. Hakimzadeh M, Behvandi Z, Valavi E, Sajadi N, Amori P. [The frequency of constipation in infants and children with urinary tract infection] [dissertation]. Ahwaz, Iran: Ahwaz Jondishapur University of Medical Sciences; 2017. Persian.

9. Asim Maqbool; Chris A. Liacouras. Functional Gastrointestinal Disorders. In: Kliegman RM, St Geme JW, Blum NJ, Shah SS, Tasker RC, Wilson KM, et al., editors. Nelson Text book of pediatrics. 21st ed. Philadelphia: Saunders Elsevier; 2019. p. 2041-5.

10. Hoque SA, Islam MT, Ahmed F, Hanif M, Islam S, Matin MA. Impact of constipation in children on urinary tract infection (UTI). Bangladesh Journal of Child Health. 2010;34(1):17-20.

11. Goldberg B, Jantausch B. Urinary Tract Infection. In: Kher KK, Greenbaum LA, Schnaper HW, editors. Clinical pediatric nephrology: Third edition. Sydney Australia: CRC Press; 2016. p. 967-91.

12. Hodsone EM, Craig JC. Urinary Tract Infections in Children. In: Avner ED, Harmon WE, Niaudet P, Yoshikawa N, Emma F, Goldstein SL, editors. Pediatric Nephrology: Seventh Edition. Berlin Heidelberg: Springer; 2016. p. 1696-719.

13. Rahhal R, Aliye UC. Functional Constipation. In: Kleinman RE, Sanderson IR, Goulet OJ, Mieli-Vergani G, Sanderson IR, Sherman PM, et al., editors. Walker's Pediatric Gastrointestinal Disease. 6th ed. Raleigh North Carolina: People's Medical Publishing House-USA; 2018. p. 2590-629.

14. Sampaio C, Sousa AS, Fraga LG, Veiga ML, Bastos Netto JM, Barroso UJ. Constipation and Lower Urinary Tract Dysfunction in Children and Adolescents: A Population-Based Study. Front Pediatr. 2016;4:101. doi: 10.3389/fped.2016.00101. [PubMed: 27752507]. [PubMed Central: PMC5046079].

15. Sarvari G, Ghane Sharbaf F, Partovi S, Elmi S, Akhavan H, Bakhtiari E. The relationship between chronic constipation and urinary tract infection in children: A case-control clinical study. International Journal of Pediatrics. 2017;5(9):5715-21.

16. Dehghani SM, Basiratnia M, Matin M, Hamidpour L, Haghighat M, Imanieh $\mathrm{MH}$. Urinary tract infection and enuresis in children with chronic functional constipation. Iranian journal of kidney diseases. 2013;7(5):363. 